\section{P79 HEALTH SERVICE USE AND ASSOCIATED COSTS ATTIBUTABLE TO DIABETES IN THE MITCHELSTOWN COHORT STUDY}

${ }^{1}$ Patrick Walsh*, ${ }^{2}$ Kate O'Neill, ${ }^{2}$ Patricia Kearney. ${ }^{1}$ School of Medicine, University College Cork, Cork, Ireland; ${ }^{2}$ School of Public Health, University College Cork, Cork, Ireland

\subsection{6/jech-2021-SSMabstracts. 167}

Background The number of people living with diabetes is increasing globally and with evidence of rising medical expenditure per person, the growth in economic burden will continue in coming years. Accurate cost of illness estimates are urgently needed to inform national policy and identify potential cost savings. With this in mind, this study aims to estimate health service use and associated costs attributable to diabetes.

Methods Diabetes was defined using self-report doctor-diagnosis, HbA1c and fasting plasma glucose levels. Health service use in the previous 12-months included; number of general practitioner (GP) visits, emergency department visits, hospital admissions, outpatient visits, and day procedures. Multivariable negative binomial regression was used to estimate the association between diabetes and frequency of visits. Frequency of visits and average marginal effects were applied to unit costs for each health service, calculating mean costs per person with and without diabetes and excess costs attributable to diabetes.

Results Out of 1,328 patients analyzed, the prevalence of diabetes was 10.5\% [95\% Confidence Interval (CI): 8.9, 12.2]. The prevalence of diagnosed diabetes was 7.4\% (95\% CI: 6.1, 8.9) and the prevalence of undiagnosed diabetes was $3.1 \%$ (95\% CI: 2.3, 4.2). In fully adjusted models, diabetes was associated with a $38 \%$ increase in GP visits. Diabetes was not associated with additional hospital admissions, emergency department visits, outpatient visits or day procedures. The annual mean cost of health service use among those with diabetes was $€ 1,620.51$ per person compared with $€ 1,340.63$ for those without. The excess health service costs attributable to diabetes were $€ 513.57$ and the national incremental healthcare costs attributable to diabetes were $€ 54,823,500.25$. Conclusion While diabetes was associated with additional GP visits, it was not associated with additional service use in secondary care. These low levels of health service utilization, among those with diabetes, may be attributable to a lack of access to endocrine services in the Munster region. It is also possible that structured diabetes management in primary care may contribute to reduced health service use and costs attributable to diabetes.

\section{P80 IMPROVING CARE FOR WOMEN WITH TYPE 1 AND TYPE 2 DIABETES AFTER A BABY LOSS: A QUALITATIVE EXPLORATION OF HEALTHCARE PROFESSIONAL AND PATIENT EXPERIENCES}

${ }^{1}$ Eleanor Dyer*, ${ }^{1}$ Ruth Bell, ${ }^{2}$ Ruth Graham, 'Judith Rankin. 'Population Health Sciences Institute, Newcastle University, Newcastle upon Tyne, UK; ${ }^{2}$ Geography, Politics and Sociology, Newcastle University, Newcastle upon Tyne, UK

\subsection{6/jech-2021-SSMabstracts. 168}

Background Women with pre-existing Type 1 and Type 2 diabetes are around four times more likely to experience baby loss: miscarriage, stillbirth, neonatal death or termination of pregnancy for medical reasons. Careful pre-pregnancy preparation can reduce the risk of loss. However, pre-pregnancy information does not talk about the challenges of becoming pregnant again after a baby has died, and uptake of pre-pregnancy care is low. Approximately 50\% of women with diabetes do not prepare for pregnancy - even after baby loss. There is a gap in understanding of how postnatal bereavement support should consider the context of diabetes and link with pre-pregnancy support for a subsequent pregnancy.

Aim To explore and better understand: (i) what it was like for women with diabetes to become pregnant again after baby loss; (ii) healthcare professional perspectives of providing prepregnancy care to this group.

Key Objective Develop recommendations for good practice to improve the support provided after a baby loss and improve preparation for future pregnancies among women with diabetes.

Methods Health professionals and women with diabetes were recruited for an interview through social media and professional networks. Recruitment is ongoing, with an anticipated sample size of 15-20 participants per group. Semi-structured interviews were used to gain in-depth experiential insights from both patient and healthcare perspectives. Interview transcripts were systematically analysed and synthesised using Thematic Analysis. Data collection and analyses are ongoing.

Results Early findings suggest individual needs differ depending on the type of diabetes and the type of loss. The influence of physical and social environments, such as living and working conditions, education level, employment status and access to transport, impact both the accessibility of care and the level of agency women with diabetes have to make the required lifestyle changes to prepare for pregnancy. Easy access to timely pre-pregnancy support from a multidisciplinary team and a sensitive discussion of pregnancy plans could reduce the risk of loss in a subsequent pregnancy. It is unclear who is responsible for supporting women in the interval between baby loss and subsequent pregnancy, and care varies across providers. Primary, community and secondary care all play a role in ensuring women with diabetes can access the care they need.

Conclusion These preliminary findings shine a new light on the challenges of becoming pregnant after a baby loss and the health service requirements for this group. Further interviews and analysis will help inform care development for women with diabetes and develop social science understanding of baby loss more generally.

\section{P81 THE ROLE OF THE SERUM METABOLOME IN DRIVING GDM IN WHITE EUROPEANS AND HIGH-RISK PAKISTANI WOMEN: A MULTIVARIATE ANALYSIS OF THE BORN IN BRADFORD COHORT}

${ }^{1}$ Harriett Fuller*, ${ }^{2,3}$ Mark lles, ${ }^{1} \mathrm{~J}$ Bernadette Moore, ${ }^{1}$ Michael Zulyniak. ${ }^{1}$ School of Food Science and Nutrition, University of Leeds, Leeds, UK; ${ }^{2}$ Leeds Institute of Medical Research, University of Leeds, Leeds, UK; ${ }^{3}$ Leeds Institute of Data Analytics, University of Leeds, Leeds, UK

\subsection{6/jech-2021-SSMabstracts. 169}

Background Women of South Asian descent are three times as likely to develop gestational diabetes mellitus (GDM) as white European (WE) mothers, independent of BMI status. This study aims to identify serum-metabolite drivers of GDM within WE and British Pakistani (PK) women from the Born in Bradford (BIB) cohort. 
Methods 146 metabolites, including dietary-related lipoproteins, fatty acids, cholesterol and amino acids, were included. Partial least squares discriminatory analyses (PLSDA) and sparse PLSDA (sPLSDA) were used to distinguish ethnic-specific metabolite signatures of GDM in 2668 WEs and 2671 PK pregnant women (gestational age $\leq 196$ days) in the BIB cohort. The impact of BMI on the metabolome and GDM risk, along with other known GDM risk factors (age, parity, multiple pregnancy and smoking status), was also examined.

Results Seven metabolites across a panel of metabolic processes (fatty acids, glycolytic, and cholesterol metabolism) were found to be predictive of GDM in both ethnicities, with fatty acids appearing to be more important drivers of GDM within WEs. Additionally, 6 metabolites were predictive of GDM solely within WEs, whilst no distinct metabolite associations were observed in PKs. Following the stratification of women by their BMI, case-status and ethnicity, a distinct metabolite profile was identified within normal-weight PK cases when compared to all other cases, characterised by a panel of amino acids and cholesterols, and glycolytic and unsaturated fatty acid metabolites.

Conclusion Serum-metabolite profiles differ by ethnicity and GDM status, largely driven by differences in fatty acid and cholesterol metabolite levels. However, in normal-weight PK women, a broad range of metabolic processes are uniquely altered and offer insight into the elevated risk of GDM observed in this otherwise healthy population. Future investigations into the determinants of these differences in metabolite profiles may shed light on the aetiology of elevated GDM risk in healthy $\mathrm{PK}$ women and direct the development of more efficacious intervention strategies.

\section{P82 REHOUSING OLDER SOCIAL HOUSING TENANTS: HEALTH-RELATED PROCESSES AND OUTCOMES}

${ }^{1}$ Stefanie Buckner*, ${ }^{1}$ Calum Mattocks, ${ }^{2}$ Lindsay Blank. ${ }^{1}$ Cambridge Public Health, University of Cambridge, Cambridge, UK; ${ }^{2}$ School of Health and Related Research, University of Sheffield, Sheffield, UK

\subsection{6/jech-2021-SSMabstracts. 170}

Background In the context of a social housing crisis, the UK must meet the housing needs of an ageing population. Rehousing schemes run by social housing providers are important means to support older (aged 55+) social housing tenants to move to appropriate homes. A better understanding of how well these schemes work is needed. This paper is based on a study (01/2020-12/2021) of the health-related processes and outcomes linked to the rehousing of older social housing tenants in Hackney/London through four local authority-supported schemes: Downsizing; Regeneration; Housing Moves; Seaside \& Country Homes.

Methods A mixed methods design entailed a scoping review, based on a search of scientific databases (MEDLINE, EMBASE, Scopus, CINAHL, PsychINFO) and the grey literature, and a narrative synthesis of results. Interviews with practice-based stakeholders across sectors $(n=11)$ were conducted. A survey of older social housing tenants who have moved through a rehousing scheme $(n=766)$, analysed in Excel, generated descriptive statistics and themes for qualitative exploration. A Photovoice component with 16 survey participants, currently underway, has been designed to capture the participants' rehousing experience and its effect on their lives. It entails interviews and focus groups. Interviews and a focus group are being conducted with older social housing tenants who were interested in being rehoused, but opted against moving ('non-movers'; $n=5$ ). All qualitative data are analysed thematically in NVivo12. All methods have been adjusted to comply with Covid-19 rules.

Results The review yielded three peer-reviewed papers and 13 reports, thus revealing a gap in knowledge around the healthrelated effects of rehousing older social housing tenants. Interviews with practice-based stakeholders highlighted promising approaches in the rehousing schemes, such as highly personalised support, and challenges, including a lack of suitable homes for tenants to move into. The survey yielded 62 valid responses (8.1\%). As well as helpful elements (e.g. being given choices) and challenges (e.g. poor communication) in the rehousing process, it identified welcome (e.g. a warmer home) and unwelcome (e.g. loneliness) health and wellbeing outcomes. The findings will be broken down by rehousing scheme. They will be supplemented by detailed insights from the Photovoice components and data collection with nonmovers.

Conclusion The study was designed to generate insights that can inform efforts by Hackney Council and other social housing providers to improve rehousing schemes for older tenants and optimise their health-related effects. Channels through which findings will be shared with decision makers and the public include a national workshop and a photo exhibition.

\section{P83 ABSTRACT WITHDRAWN}

\section{P84 ABSTRACT WITHDRAWN}

\section{P85 HOW TO TACKLE UNINTENTIONAL DISCRIMINATION IN PRIMARY HEALTH CARE: GENERAL PRACTITIONERS' IMPLICIT BIASES AND CULTURAL COMPETENCE}

Camille Duveau*, Vincent Lorant. Institute of Research Health and Society, University Catholic of Louvain, Brussels, Belgium

\subsection{6/jech-2021-SSMabstracts. 171}

Background Reducing ethnic inequalities in health care is a worldwide priority. General practice is no exception to this, as studies pointed to ethnic discrimination in diagnosis, referral and treatment of racial minorities patients by General Practitioners (GPs). However, it is unclear whether these discriminations are due to implicit bias or by the lack of explicit cultural competences, two explanations demanding different intervention. Implicit biases are a human trait which can influence stereotypes and care behaviour whereas cultural competence is 'the ability to work and communicate effectively and appropriately with people from culturally different backgrounds'.

Methods This mixed-method study investigates the existence of implicit biases and the level of cultural competences among GPs trainees. Data collection among GPs trainees (2021) was used and analysed in SAS Enterprise Guide. The 170 